\title{
Hydro-morphodynamics 2D modelling using a discontinuous Galerkin discretisation
}

\author{
Mariana C. A. Clare ${ }^{a, *}$, James R. Percival ${ }^{a}$, Athanasios Angeloudis ${ }^{b}$, Colin J. Cotter $^{c}$ and \\ Matthew D. Piggott ${ }^{a}$ \\ ${ }^{a}$ Department of Earth Science and Engineering, Imperial College London, UK. \\ ${ }^{b}$ School of Engineering, Institute for Infrastructure \& Environment, University of Edinburgh, UK. \\ ${ }^{c}$ Department of Mathematics, Imperial College London, UK.
}

\section{This manuscript is a non-peer reviewed EarthArXiv preprint that has been submitted for publication in COMPUTERS \& GEOSCIENCES.}

\section{Introduction}

Data from 2010 shows that almost 400 million people lived in areas less than 5m above average sea level (CIESIN, 2013) and this population keeps growing. As sea levels rise and with the potential for storms to increase in strength and frequency due to a changing climate, the coastal zone is becoming an ever more critical location for the application of advanced modelling techniques. A particularly important example is the development and application of improved morphodynamic models to simulate sediment transport accurately. The effects of climate change will cause hydrodynamic changes leading to increased erosion rates, increasing flooding and erosion risk in coastal zones. The coupled and non-linear nature of this problem makes it especially challenging, since models must solve both hydrodynamic and sediment trsnsport processes together with their two-way coupled interactions. Furthermore, there are two types of sediment transport processes that should be resolved: suspended sediment in the fluid and bedload transport propagating along the bed itself.

Over the last 40 years, increasingly complex morphodynamic models have been developed to predict sediment transport in fluvial and coastal zones. These models can be one-dimensional (1D), two-dimensional (2D) or threedimensional (3D), and are discussed in detail in Amoudry (2008), Amoudry and Souza (2011) and Papanicolaou et al. (2008), which we draw upon for a brief review here. 1D models generally use finite difference methods to solve a simple system of equations and are the cheapest computationally. However, they cannot capture velocity in the crossstream and vertical directions. 2D (or 2DH) models adopt the shallow water approximation and can use finite difference (e.g. XBeach - Roelvink et al., 2015), finite volume (e.g. Mike 21 - Warren and Bach, 1992), or finite element based methods to solve a more complex system of equations. They capture velocity in both the streamwise and cross-stream directions on planview geometries in the horizontal. 3D models are similar to 2D, but solve an even more complex full system of equations using finite difference (e.g. ROMS - Warner et al., 2008), finite volume (e.g. Fast3d Landsberg et al., 1998) or finite element based methods. They are thus potentially more accurate, but considerably more computationally expensive. More sophisticated models offer 2D and 3D options, such as Telemac-Mascaret (Hervouet, 1999) and Delft3d (Deltares, 2014), which use finite element/volume and finite difference based methods, respectively. In choosing a model, one must balance the simplicity and computational efficiency of a 2D model against the potential accuracy of a 3D one.

\footnotetext{
Author credits

Mariana C. A. Clare: Code development and verification, experimental design, manuscript preparation. James R. Percival: Technical supervision, manuscript review and editing. Athanasios Angeloudis: Technical supervision, manuscript review and editing. Colin J. Cotter: Project supervision, manuscript review and editing. Matthew D. Piggott: Project supervision, manuscript review and editing.
}

\section{Email addresses}

@m.clare17@imperial.ac.uk (M.C.A.Clare); j.percival@imperial.ac.uk(J.R. Percival); A.Angeloudis@ed.ac.uk (A. Angeloudis); colin.cotter@imperial.ac.uk (C.J. Cotter); m.d.piggott@imperial.ac.uk (M.D. Piggott) 
Despite this variety of approaches, Syvitski et al. (2010) argue the need for more accurate and faster morphodynamic models. The aim of this work is to present a novel and flexible 2D depth-averaged coupled hydrodynamic and sediment transport model developed within Thetis, a finite element coastal ocean modelling system (Kärnä et al., 2018) built using the Firedrake code generation framework (Rathgeber et al., 2017). This framework is versatile and ensures our underlying code is robust and optimised, and can be executed efficiently in parallel. Furthermore, it means our model is easily extensible and further work could include using an adjoint allowing a sensitivity analysis to be conducted (Farrell et al., 2013) or using an adaptive mesh resulting in a decrease in computational cost (McManus et al., 2017).

In this work, a 2D model is an appropriate choice because the depth-scale is much smaller than the horizontal for the cases discussed. We extend Thetis' existing capability to model scalar transport to a capacity to model suspended sediment transport and add within it a new capability to model bedload transport and bedlevel changes. For validation purposes, we compare our results with experimental data and Telemac-Mascaret's 2D model (Hervouet, 1999), which is widely-used (Amoudry and Souza, 2011; Papanicolaou et al., 2008). We improve on existing state-of-the-art models by using a discontinuous Galerkin based finite element discretisation (DG) available in Thetis (Kärnä et al., 2018). DG has several advantages including being locally mass conservative, meaning sediment is conserved on an element-by-element level, which is an advantage for coupling (Dawson, Sun and Wheeler, 2004); being well-suited to advection-dominated problems like ours (Kärnä et al., 2018); being geometrically flexible; and allowing higher order local approximations (Li, 2006). Morphodynamic models using DG have been presented in Kubatko, Westerink and Dawson (2006), Michoski et al. (2013) and Tassi et al. (2008), but without suspended sediment transport. To the best of our knowledge, our model is the first morphodynamic model with both bedload and suspended sediment transport to use DG.

The remainder of this paper is structured as follows: in Section 2 we describe our coupled hydrodynamic and sediment transport model; in Section 3 we explore the finite element model Thetis; in Sections 4 and 5, we use the test cases of a migrating trench and a meander to validate our model; in Section 6 we benchmark our test cases against Sisyphe and finally in Section 7 conclude this work.

\section{Model derivation}

\subsection{Hydrodynamic and sediment transport equations}

In this subsection, we describe the general equations for modelling the hydrodynamic and sediment transport flow, and follow the presentation and notation of $\mathrm{Wu}$ (2007), where more details can be found.

The hydrodynamic component of the sediment-water mixture is governed by the (3D) Navier-Stokes equations for single phase flow. We use the $2 \mathrm{D}$ version of Thetis assuming the only external force acting on the system is gravity. We also assume any wavelength is much longer than the depth of the fluid, hence the vertical flow variation is small enough to be negligible and $\partial u_{1} / \partial z=\partial u_{2} / \partial z=0$ (for more details, see Segur, 2009).

The 2D model is derived by depth-averaging from the bed, $z_{b}$, to the water surface, $\eta$, the hydrodynamic equations. Thus, we must define conditions at the fluid boundaries: we apply the kinematic boundary condition at $\eta$ as a free moving boundary, and we consider $z_{b}$ to be impermeable. Since the bed evolution is slow, imposing a no-slip condition at $z_{b}$ means $u_{1}=u_{2}=0$ here and thus the simplified depth-averaged equation for the conservation of mass is

$$
\frac{\partial \eta}{\partial t}+\frac{\partial}{\partial x}\left(h \bar{u}_{1}\right)+\frac{\partial}{\partial y}\left(h \bar{u}_{2}\right)=0
$$

where $h=\eta-z_{b}$ is the depth, and $\bar{u}_{1}$ and $\bar{u}_{2}$ are the depth-averaged velocities in the $x$ and $y$ directions, respectively. Note that following convention, depth-averaged variables are denoted with an overbar, as $\overline{-}$

Applying the boundary conditions, combining dispersion and stress effects, and assuming there is no wind-driving force on the water surface, the simplified depth-averaged equation for the conservation of momentum is

$$
\frac{\partial\left(h \bar{u}_{i}\right)}{\partial t}+\frac{\partial\left(h \overline{u_{1} u_{i}}\right)}{\partial x}+\frac{\partial\left(h \overline{u_{2} u_{i}}\right)}{\partial y}=-g h \frac{\partial \eta}{\partial x_{i}}+\frac{1}{\rho} \frac{\partial\left(h \overline{\tau_{i 1}}\right)}{\partial x}+\frac{1}{\rho} \frac{\partial\left(h \overline{\tau_{i 2}}\right)}{\partial y}-\frac{\tau_{b i}}{\rho},
$$

where, following the notation of $\mathrm{Wu}(2007), \bar{\tau}_{i j}=\mu_{t}\left(\frac{\partial \bar{u}_{i}}{\partial x_{j}}+\frac{\partial \bar{u}_{j}}{\partial x_{i}}\right)$ and $\mu_{t}$ is the dynamic eddy viscosity. Note that $i=1,2$ represents the $x, y$-direction. Eq. (1) and (2) comprise the hydrodynamic component of our model. 
We take an Eulerian approach for the sediment transport equations, rather than the more computationally expensive Lagrangian approach, and make a macroscopic assumption. We thus represent the sediment dynamics via an advectiondiffusion equation for a sediment concentration field, $c$. Note that in this work we only consider non-cohesive sediment.

If the sediment diameter is finer than $1 \mathrm{~mm}$ and the sediment concentration, $c$, is lower than $10 \%$ of the fluid volume then we can assume there is no mixing at the 'molecular level'. Hence, there is no diffusion and the only significant relative motion between the flow and the sediment is settling due to gravity. Furthermore, the low concentration and fine sediment size means the settling velocity of the sediment particles $w_{s}$ can be approximated by that of a single sediment particle in clear water. The equation governing the sediment concentration is

$$
\frac{\partial c}{\partial t}+\frac{\partial\left(u_{j} c\right)}{\partial x_{j}}=\frac{\partial}{\partial x_{j}}\left(w_{s} c \delta_{3 j}\right)
$$

where $\delta_{3 j}$ is the Kronecker delta applied to the vertical component. Time-averaging Eq. (3) to filter turbulence introduces a diffusivity term, $\epsilon_{s} \frac{\partial c}{\partial x_{j}}$, and becomes

$$
\frac{\partial c}{\partial t}+\frac{\partial\left(u_{1} c\right)}{\partial x}+\frac{\partial\left(u_{2} c\right)}{\partial y}+\frac{\partial\left(u_{3} c\right)}{\partial z}-\frac{\partial\left(w_{s} c\right)}{\partial z}=\frac{\partial}{\partial x}\left(\epsilon_{s} \frac{\partial c}{\partial x}\right)+\frac{\partial}{\partial y}\left(\epsilon_{s} \frac{\partial c}{\partial y}\right)+\frac{\partial}{\partial z}\left(\epsilon_{s} \frac{\partial c}{\partial z}\right)
$$

where $\epsilon_{S}$ is the so-called sediment turbulent diffusivity coefficient, which can be chosen to take a larger than physically realistic value as an approximation for unresolved turbulence effects.

As bedload transport occurs along the bed and suspended sediment transport occurs across the fluid water column, the domain is conceptually divided into bedload and suspended sediment zones with an interface at $z=z_{b}+\delta$ consistent with Tassi and Villaret (2014). At this interface, we define a gradient boundary condition of $E_{b}=-\left.\epsilon_{s} \frac{\partial c}{\partial z}\right|_{z=z_{b}+\delta}=$ $w_{s} c_{b *}$ and $D_{b}=w_{s} c_{b}$, where $E_{b}$ is the near-bed sediment erosion flux, $D_{b}$ the deposition flux. As $\delta$ is assumed to be small, following standard practice, the boundary condition is applied at $z=z_{b}$. Therefore, depth-averaging Eq. (4), using the boundary conditions, combining the diffusion and dispersion effects, and recalling we are modelling a long-term sedimentation process, we obtain

$$
\frac{\partial}{\partial t}(h \bar{c})+\frac{\partial}{\partial x}\left(h \overline{u_{1} c}\right)+\frac{\partial}{\partial y}\left(h \overline{u_{2} c}\right)=\frac{\partial}{\partial x}\left[h\left(\epsilon_{s} \frac{\partial \bar{c}}{\partial x}\right)\right]+\frac{\partial}{\partial y}\left[h\left(\epsilon_{s} \frac{\partial \bar{c}}{\partial y}\right)\right]+E_{b}-D_{b} .
$$

Due to the coupled nature of our model, we cannot calculate $\overline{\mathbf{u} c}$, but only the product of $\overline{\mathbf{u}}$ (from the hydrodynamic component) and $\bar{c}$ (from the sediment transport component). These two quantities are not equal because the product of two integrated variables is not equal to the integral of their product. Thus, following Huybrechts, Villaret and Hervouet (2010), we rewrite Eq. (5) as an advection-diffusion equation for $\bar{c}$

$$
\frac{\partial}{\partial t}(h \bar{c})+\frac{\partial}{\partial x}\left(h u_{\mathrm{adv}_{1}} \bar{c}\right)+\frac{\partial}{\partial y}\left(h u_{\mathrm{adv}_{2}} \bar{c}\right)=\frac{\partial}{\partial x}\left[h\left(\epsilon_{s} \frac{\partial \bar{c}}{\partial x}\right)\right]+\frac{\partial}{\partial y}\left[h\left(\epsilon_{s} \frac{\partial \bar{c}}{\partial y}\right)\right]+E_{b}-D_{b}
$$

with advection velocity

$$
\mathbf{u}_{\mathrm{adv}}=\frac{\overline{\mathbf{u} c}}{\bar{c}}
$$

We then use a correction factor $F_{\text {corr }}=\mathbf{u}_{\text {adv }} / \overline{\mathbf{u}}$ to convert $\overline{\mathbf{u}}$ into $\mathbf{u}_{\text {adv }}$. Continuing to follow Huybrechts, Villaret and Hervouet (2010), if we assume $\mathbf{u}$ has a logarithmic profile and $c$ has a Rouse concentration profile, we obtain

$$
F_{\text {corr }}=\frac{I_{2}-\log \left(\frac{B}{30}\right) I_{1}}{I_{1} \log \left(\frac{e B}{30}\right)}
$$

where

$$
I_{1}=\int_{B^{-1}}^{1}\left(\frac{(1-a)}{a}\right)^{R} d a
$$




$$
I_{2}=\int_{B^{-1}}^{1} \log a\left(\frac{(1-a)}{a}\right)^{R} d a
$$

with a $=z / h, \mathrm{~B}=h / k_{s}^{\prime}$, where $k_{s}^{\prime}=3 d_{50}$ is the grain roughness coefficient, and $R=w_{s} / \kappa u_{*}$ the Rouse number, where $\kappa$ the Von Kármán constant (given as 0.4 in Wu, 2007) and $u_{*}$ the shear velocity. To avoid numerical integration, the Rouse concentration profile is simplified, such that Eq. (9) becomes

$$
\begin{gathered}
I_{1}= \begin{cases}\frac{1}{1-R}\left(1-B^{1-R}\right), & R \neq 1, \\
-\log (B), & R=1,\end{cases} \\
I_{2}= \begin{cases}\frac{I_{1}+\log (B) B^{1-R}}{R-1}, & R \neq 1, \\
-0.5(\log (B))^{2}, & R=1 .\end{cases}
\end{gathered}
$$

Finally, the sediment concentration equation is

$$
\frac{\partial}{\partial t}(h \bar{c})+\frac{\partial}{\partial x}\left(h F_{\text {corr }} \bar{u}_{1} \bar{c}\right)+\frac{\partial}{\partial y}\left(h F_{\text {corr }} \bar{u}_{2} \bar{c}\right)=\frac{\partial}{\partial x}\left[h\left(\epsilon_{s} \frac{\partial \bar{c}}{\partial x}\right)\right]+\frac{\partial}{\partial y}\left[h\left(\epsilon_{s} \frac{\partial \bar{c}}{\partial y}\right)\right]+E_{b}-D_{b} .
$$

\subsection{Suspended Sediment Transport}

To fully describe Eq. (11), we calculate the sediment source term, $E_{b}-D_{b}$, where $E_{b}$ is the erosion flux and $D_{b}$ the deposition flux. From the gradient boundary condition, we recall that

$$
E_{b}-D_{b}=w_{s} c_{b *}-w_{s} c_{b}=w_{s} c_{b *}-w_{s} \alpha_{c} \bar{c},
$$

where $w_{s}$ is the settling velocity of the particles, $c_{b *}$ the equilibrium near-bed sediment concentration, $c_{b}=\alpha_{c} \bar{c}$ the actual near-bed sediment concentration, and $\alpha_{c}$ a coefficient greater than 1 which accounts for the near-bed sediment concentration value being higher than $\bar{c}$ due to gravity. We choose to approximate $\alpha_{c}$ using the following formula derived in Tassi and Villaret (2014),

$$
\frac{1}{\alpha_{c}}= \begin{cases}\left|\frac{A\left(1-A^{r}\right)}{r}\right|, & |R-1|>10^{-4}, \\ |-A \log (A)|, & |R-1| \leq 10^{-4},\end{cases}
$$

where

$$
r= \begin{cases}\min (R-1,3), & |R-1|>10^{-4} \\ 0, & |R-1| \leq 10^{-4}\end{cases}
$$

$A=\max \left(\frac{\delta}{h}, 1\right), R$ the Rouse number, and $\delta$ the height of the bedload zone.

We calculate $w_{s}$ in Eq. (12) using the formula defined in Van Rijn (1984)

$$
w_{s}= \begin{cases}\frac{g \Delta d_{50}^{2}}{18 v}, & d_{50} \leq 10^{-4}, \\ \frac{10 v}{d_{50}}\left(\sqrt{1+0.01 \frac{g \Delta d_{50}^{3}}{v^{2}}}-1\right), & 10^{-4} \leq d_{50} \leq 10^{-3}, \\ 1.1 \sqrt{g \Delta d_{50}}, & d_{50}>10^{-3},\end{cases}
$$

where $d_{50}$ is the median sediment diameter, $v$ the kinematic molecular viscosity, and

$$
\Delta=\frac{\rho_{s}}{\rho_{f}}-1,
$$

where $\rho_{s}$ is the sediment density, and $\rho_{f}$ the water density. 
As discussed in Garcia and Parker (1991), there are alternative formulae for $c_{b *}$ in Eq. (12). Here we use the van Rijn formula which is applicable for fine sediments when no waves are present and is given in Van Rijn (1984) as

$$
c_{b *}=0.015 \frac{d_{50}}{\delta} \frac{S_{0}^{3 / 2}}{d_{*}^{3 / 10}},
$$

where $d_{*}$ is the non-dimensional diameter

$$
d_{*}=d_{50}\left(\frac{g \Delta}{v^{2}}\right)^{1 / 3},
$$

and $S_{0}$ the transport stage parameter

$$
S_{0}=\frac{\Psi \tau_{b}-\tau_{c}}{\tau_{c}} .
$$

See Tassi and Villaret (2014) for more detail. In Eq. (19), $\tau_{c}$ is the critical shear stress

$$
\tau_{c}=\left(\rho_{s}-\rho_{f}\right) g d_{50} \theta_{c r}
$$

where $\theta_{c r}$ is the critical shields parameter; $\tau_{b}$ is the bed shear stress acting against the velocity flow and equal in magnitude in both directions

$$
\tau_{b}=\frac{1}{2} \rho_{f} C_{h}\left(\bar{u}_{1}^{2}+\bar{u}_{2}^{2}\right)
$$

where $\left(\bar{u}_{1}, \bar{u}_{2}\right)$ is the depth-averaged velocity; and $\Psi$ is the skin friction correction

$$
\Psi=\frac{2 \kappa^{2}}{C_{h}\left[\log \left(\frac{11.036 h}{k_{s}^{\prime}}\right)\right]^{2}},
$$

where $C_{h}$ is the Nikuradse quadratic drag coefficient

$$
C_{h}=2 \frac{\kappa^{2}}{\log \left(\frac{11.036 h}{k_{s}}\right)^{2}},
$$

where $k_{s}$ is the Nikuradse friction height.

The actual skin friction is implemented using Eq. (23) but with $k_{s}$ replaced by $k_{s}^{\prime}$ (as in Tassi and Villaret, 2014).

\subsection{Bedload transport}

Following Tassi and Villaret (2014), for modelling bedload transport we define the bedload transport flux, $\mathbf{Q}_{\mathbf{b}}$

$$
\mathbf{Q}_{\mathbf{b}}=\phi_{s} \sqrt{g\left(\frac{\rho_{s}}{\rho_{f}}-1\right) d_{50}^{3}}(\cos \xi, \sin \xi),
$$

where $\cos \xi=\frac{\bar{u}_{1}}{\sqrt{\bar{u}_{1}^{2}+\bar{u}_{2}^{2}}}$ and $\sin \xi=\frac{\bar{u}_{2}}{\sqrt{\bar{u}_{1}^{2}+\bar{u}_{2}^{2}}}$. We choose the Meyer-Peter-Müller formula to define the non-dimensional sediment rate $\phi_{S}$

$$
\phi_{s}= \begin{cases}0, & \theta^{\prime}<\theta_{\mathrm{cr}} \\ \alpha_{\mathrm{MPM}}\left(\theta^{\prime}-\theta_{\mathrm{cr}}\right)^{3 / 2}, & \text { otherwise }\end{cases}
$$

where $\theta_{\mathrm{cr}}$ is the critical shields parameter, $\alpha_{\mathrm{MPM}}$ a coefficient equal to 8, as suggested by Tassi and Villaret (2014), and $\theta^{\prime}$ the non-dimensional shields parameter

$$
\theta^{\prime}=\frac{\Psi \tau_{b}}{\left(\rho_{s}-\rho_{f}\right) g d_{50}},
$$

with $\Psi$ given by Eq. (19) and $\tau_{b}$ by Eq. (21). 


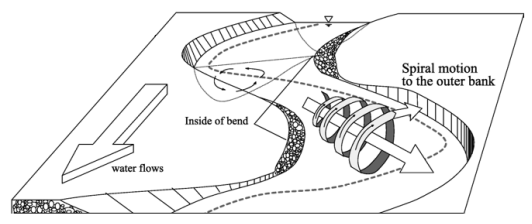

Figure 1: Secondary current in curved channel, adapted from Park and Ahn (2019).

\subsubsection{Slope effect}

In practice, the magnitude and direction of $\mathbf{Q}_{\mathbf{b}}$ depends on the gradient of the bed, but this is not reflected in Eq. (24). When the bed has a positive gradient in the transport direction, gravity acts against the sediment causing the magnitude of $\mathbf{Q}_{\mathbf{b}}$ to decrease and its direction to alter, and vice versa for a negative gradient.

(i) Magnitude correction

To correct the magnitude we use

$$
\mathbf{Q}_{\mathbf{b} *}=\mathbf{Q}_{\mathbf{b}}\left(1-\Upsilon \frac{\partial z_{b}}{\partial s}\right)
$$

given in Soulsby (1997), where $s$ is a direction tangential to the current and $\Upsilon$ an empirical coefficient set to 1.3 (Tassi and Villaret, 2014).

(ii) Angle correction

To correct the angle, following Talmon, Struiksma and Mierlo (1995), we set

$$
T=\frac{1}{\beta_{2} \sqrt{\theta}},
$$

where $\beta_{2}$ is an empirical coefficient (equal to 1.5 for river test cases) and $\theta$ is given by

$$
\theta=\frac{\left(\rho_{f}-\rho_{s}\right) g d_{50}}{\max \left(\frac{1}{2} \rho_{f} C_{h}\|\overline{\mathbf{u}}\|^{2}, 10^{-10}\right)},
$$

with $C_{h}$ defined as in Eq. (23). Thus

$$
\mathbf{Q}_{\mathbf{b}}=\left(\phi_{s} \sqrt{g\left(\frac{\rho_{s}}{\rho_{f}}-1\right) d_{50}^{3}}(\cos \alpha, \sin \alpha)\right),
$$

where $\alpha$ is the corrected angle defined by

$$
\left(\begin{array}{c}
\sin \alpha \\
\cos \alpha
\end{array}\right)=\frac{1}{\|\mathbf{p}\|^{2}}\left(\begin{array}{c}
p_{1} \\
p_{2}
\end{array}\right)=\frac{1}{\|\mathbf{p}\|^{2}}\left(\begin{array}{l}
\sin \xi-T\left(\frac{\partial z_{b}}{\partial y}\right) \\
\cos \xi-T\left(\frac{\partial z_{b}}{\partial x}\right)
\end{array}\right),
$$

where $\mathbf{p}=\left(p_{1}, p_{2}\right)$.

\subsubsection{Secondary current}

As shown in Figure 1, depth-averaged models for curved channels need to account for both the current and a helical flow effect. This affects the magnitude and direction of $\mathbf{Q}_{\mathbf{b}}$ and in Tassi and Villaret (2014) is implemented on top of slope effect corrections. Therefore, we implement a secondary current using

$$
\tan \varsigma=7 \frac{h}{r},
$$

given in Engelund (1974), where $\varsigma$ is the angle between the bedload transport and the main flow direction, $h$ the mean water depth, and $r$ the local radius of curvature of the streamline calculated using

$$
r=\frac{\alpha^{\prime}\left(\bar{u}_{1}^{2}+\bar{u}_{2}^{2}\right)}{g \frac{\partial \eta}{\partial n}}
$$


where $\eta$ is the elevation, $n$ a direction normal to the current and $\alpha^{\prime}$ a coefficient which lies between 0.75 (rough bed) and 1 (smooth bed).

Using Eq. (32), we construct the term

$$
\Xi=\sqrt{\left(\tau_{b} \Upsilon \cos \alpha+\tau_{b} \bar{u}_{2} \tan \varsigma\right)^{2}+\left(\tau_{b} \Upsilon \sin \alpha-\tau_{b} \bar{u}_{1} \tan \varsigma\right)^{2}},
$$

where $\tau_{b}$ is the bedload shear stress defined by Eq. (21), and $\alpha$ and $\Upsilon$ are the corrected flow angle and magnitude factors (Section 2.3.1).

Hence, we define a new corrected bed transport flow direction $\hat{\alpha}$ with

$$
\begin{aligned}
\cos \hat{\alpha} & =\frac{\tau_{b} \Upsilon \cos \alpha+\tau_{b} \bar{u}_{2} \tan \varsigma}{\Xi}, \\
\sin \hat{\alpha} & =\frac{\tau_{b} \Upsilon \sin \alpha-\tau_{b} \bar{u}_{1} \tan \varsigma}{\Xi},
\end{aligned}
$$

and a new slope magnitude correction factor

$$
\hat{\Upsilon}=\frac{\Xi}{\tau_{b}} .
$$

Note that if a secondary current effect is imposed without slope effect corrections, then $\Upsilon=1$ and $\alpha=\xi$, i.e. the original flow angle.

\subsection{Calculating the new bedlevel}

The new bedlevel, $z_{b}$, is affected by both the suspended sediment and bedload transport described in Sections 2.2 and 2.3, and is governed by the Exner equation

$$
\left(1-p^{\prime}\right) \frac{d z_{b}}{d t}+\nabla_{h} \cdot \mathbf{Q}_{\mathbf{b}}=D_{b}-E_{b}
$$

where $p^{\prime}$ is the bed sediment porosity. This completes the model equations.

\subsection{Practical application within the Thetis framework}

When implementing our model, we use two common techniques for algorithm stability and efficiency reasons.

\subsubsection{Spinning up the hydrodynamics}

Once the simulation starts, we are forcing a previously motionless flow, and the resulting flow instabilities could trigger unrealistic bedlevel changes. Following standard practice (e.g. Gerritsen et al., 2008), we avoid this by first running a simulation solving only the hydrodynamic equations. When the velocity and elevation fields have reached an approximate steady state, we introduce sediment and enable bedlevel changes.

\subsubsection{Morphological scale factor}

Once running a bed evolution simulation for a long period of time, a morphological scale factor, $m$, is often used (e.g. Gerritsen et al., 2008) which increases the rate of bedlevel changes and saves computational time. This factor means that each $\Delta t$ in the hydrodynamic and sediment concentration equations is equivalent to $m \Delta t$ for the bed evolution. We implement this by including the factor $m$ in the Exner equation (37)

$$
\frac{\left(1-p^{\prime}\right)}{m} \frac{d z_{b}}{d t}+\nabla_{h} \cdot \mathbf{Q}_{\mathbf{b}}=D_{b}-E_{b}
$$

This factor is suitable because the hydrodynamics are in an approximate steady state, and we assume throughout that changes in the bed are significantly slower than in the hydrodynamics. 


\section{Finite element based implementation}

We build on existing elements of Thetis for the implementation of a coupled hydrodynamic and sediment transport model. Thetis is a finite element coastal ocean modelling system (built using the code generating framework Firedrake) which is first described in Kärnä et al. (2018) with a 3D model. We use the 2D depth-averaged version of Thetis outlined in Vouriot et al. (2019), which solves the shallow water equations and the non-conservation form of a depth-averaged sediment concentration equation, as discussed in the previous section.

We use a discontinuous Galerkin based finite element discretisation (DG) which has several advantages in this context, as discussed in Section 1.

\subsection{DG based methods in Thetis}

When using DG based methods, we generate an unstructured mesh of triangular elements tesselating our domain $\Omega$ and then define our finite element space on this mesh. Using a discontinuous function space requires the definition of variables on element edges (including on the domain boundary $d \Omega$ ), with the union of these edges denoted by $\Gamma$. The average operator $\{\{\cdot\}\}$ and jump operator $[[\cdot]]$ across the interior edges on scalar and vector fields are

$$
\begin{gathered}
\{\{\mathbf{X}\}\}=\frac{1}{2}\left(\mathbf{X}^{+}+\mathbf{X}^{-}\right), \quad[[\chi]]_{\mathbf{n}}=\chi^{+} \mathbf{n}^{+}+\chi^{-} \mathbf{n}^{-}, \\
{[[\mathbf{X}]]_{\mathbf{n}}=\mathbf{X}^{+} \cdot \mathbf{n}^{+}+\mathbf{X}^{-} \cdot \mathbf{n}^{-},}
\end{gathered}
$$

where $\mathbf{n}=\left(n_{x}, n_{y}, 0\right)$ is the horizontal projection of the outward pointing unit normal on the element edge, and ' + ' and '-' denote either side of the interior edge.

\subsubsection{Depth-averaged sediment concentration equation}

Thetis uses very similar techniques to solve the hydrodynamic equations, (1) and (2), and the sediment concentration equation (11). We focus on the latter because it is the most pertinent for this work; the formulation for the hydrodynamic equations can be found in Kärnä et al. (2018), Pan, Kramer and Piggott (2019) and Vouriot et al. (2019). In the advection term, Thetis uses an upwinding scheme for the sediment concentration, $\bar{c}$ : at each edge, $\bar{c}$ is chosen to be equal to its upstream value with respect to velocity, $\bar{c}^{\text {up }}$ (see Leveque, 1996). Hence, the weak form of the advection term $\overline{\mathbf{u}} \cdot \nabla_{h} \bar{c}$ in Eq. (11) is

$$
\int_{\Omega} \psi \overline{\mathbf{u}} \cdot \nabla_{h} \bar{c} d x=-\int_{\Omega} \bar{c} \nabla_{h} \cdot(\overline{\mathbf{u}} \psi) d x+\int_{\Gamma} \bar{c}^{\mathrm{up}}[[\psi \overline{\mathbf{u}}]]_{\mathbf{n}} d s .
$$

Following Kärnä et al. (2018), we must apply a stabilisation method to the weak form of the diffusivity term, $-\nabla_{h}$. $\left(\epsilon_{s} \nabla_{h} \bar{c}\right)$, to ensure the discretisation is stable for elliptic operators. We use the Symmetric Interior Penalty Galerkin (SIPG) method given in Epshteyn and Rivière (2007), with the penalty parameter $\sigma$ given in Kärnä et al. (2018). Hence, the weak form of the diffusivity term is

$-\int_{\Omega} \psi \nabla_{h} \cdot\left(\epsilon_{s} \nabla_{h} \bar{c}\right) d x=\int_{\Omega} \epsilon_{s}\left(\nabla_{h} \psi\right) \cdot\left(\nabla_{h} \bar{c}\right) d x-\int_{\Gamma}[[\psi]]_{\mathbf{n}} \cdot\left\{\left\{\epsilon_{s} \nabla_{h} \bar{c}\right\}\right\} d s-\int_{\Gamma}[[\bar{c}]]_{\mathbf{n}} \cdot\left\{\left\{\epsilon_{s} \nabla_{h} \psi\right\}\right\} d s+\int_{\Gamma} \sigma\left\{\left\{\epsilon_{s}\right\}\right\}[[\bar{c}]]_{\mathbf{n}} \cdot[[\psi]]_{\mathbf{n}} d s$.

The use of upwinded numerical fluxes and slope limiters makes our model robust for modelling the steep bed gradients formed (see Kubatko, Westerink and Dawson, 2006). Finally, the weak form of the sediment transport source term, $E_{b}-D_{b}$, is simply

$$
\int_{\Omega}(\text { Source Term }) \psi d x=\int_{\Omega}\left(E_{b}-D_{b}\right) \psi d x
$$

We use the implicit backward Euler method to proceed from the $n^{\text {th }}$ to the $(n+1)^{t h}$ timestep in Eq. (11). Thus, the final equation is

$$
\int_{\Omega} \frac{\bar{c}_{i}^{(n+1)}-\bar{c}_{i}^{(n)}}{\Delta t} \psi d x=F_{i}^{(n+1)},
$$

where $F_{i}^{(n+1)}$ is the sum of the weak forms (39), (40) and (41). The combination of a DG based method with an implicit timestepping method makes our model more robust. 


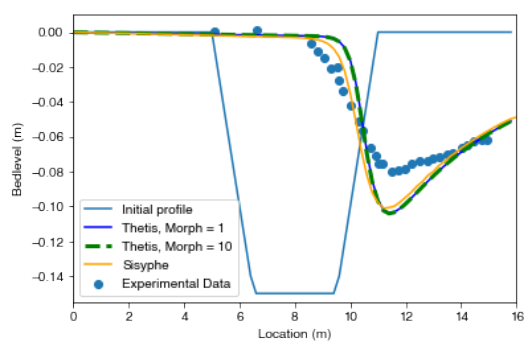

Figure 2: Bedlevel after $15 \mathrm{~h}$ for different morphological scale factors comparing experimental data, Sisyphe and Thetis with $\Delta t=$ 0.05 s. Experimental data and initial trench profile source: Villaret et al. (2016).

\subsubsection{Exner Equation}

In order to avoid grid-scale noise and unstable oscillations when solving the Exner equation (37), we define the bedlevel, $z_{b}$, on a continuous grid, and thus use a continuous Galerkin based finite element discretisation (CG). We project all hydrodynamic and sediment transport variables from the DG space into the CG space before calculating the terms in the Exner equation. This causes a very slight loss of accuracy in our model variables, but overall a more stable bedlevel result. The weak form of the divergence term $\nabla_{h} \cdot \mathbf{Q}_{\mathbf{b}}$ is

$$
\int_{\Omega} \psi \nabla_{h} \cdot \mathbf{Q}_{\mathbf{b}} d x=-\int_{d \Omega}\left(\mathbf{Q}_{\mathbf{b}} \cdot \mathbf{n}\right) \psi d s+\int_{\Omega}\left(\mathbf{Q}_{\mathbf{b}} \cdot \nabla_{h}\right) \psi d x
$$

On a continuous grid and assuming centred fluxes on interior edges, the values on either side of each interior edge cancel over the whole domain, meaning that the only boundary contribution is from the domain boundary, $d \Omega$. We use the implicit backward Euler method to solve Eq. (37) allowing us to use large timesteps stably. Thus

$$
\int_{\Omega}\left(\left(1-p^{\prime}\right) \frac{z_{b_{i}}^{(n+1)}-z_{b_{i}}^{(n)}}{\Delta t}\right) \psi d x=G_{i}^{(n+1)}
$$

where $G_{i}^{(n+1)}$ is the sum of the weak forms (41) and (43). Note that the radius of curvature, (33), in the secondary current parametrisation is dependent on the surface elevation $\eta$ rather than on $z_{b}$. Hence, we rewrite $\eta$ as $\left(h+z_{b}\right)$ meaning we can benefit from an implicit discretisation.

\section{Migrating trench test case}

In this section, we consider the simple test case of a migrating trench (as in, for example, Gerritsen et al. 2008 and Van Rijn 1980). We use this test case to validate the implementation of the mathematical and numerical methods used in Thetis by using experimental data from a lab study in Van Rijn (1980) and results from Villaret et al. (2016).

In Villaret et al. (2016), for this test case, a coupled model is used comprising of Telemac-Mascaret's 2D depthaveraged hydrodynamic module, Telemac2D, and its sediment transport and bed evolution module, Sisyphe. Hereafter in this work, we refer to this coupled model as Sisyphe. For the discretisation, they use Telemac-Mascaret's continuous finite element model (Danilov, 2013) with the method of characteristics for the hydrodynamic advection terms and distributive schemes for the sediment transport advection terms. The method of characteristics has the advantage of being unconditionally stable, but is not mass conservative and is diffusive for small timesteps, meaning the problem is artificially regularised with potentially spurious mixing. Distributive schemes are mass conservative, but also have high numerical diffusion and Courant number limitations to ensure stability. For further details on both methods, see Hervouet (2007) and Tassi and Villaret (2014). The limitations of these two methods in part motivate our use of DG based methods in Thetis.

\subsection{Test case configuration}

In Figure 2, the initial trench profile and the final bedlevel profile after a $15 \mathrm{~h}$ experiment is observed demonstrating the trench migration over time.

For Sisyphe, we use the model of Villaret et al. (2016), and summarise the parameter values in Table 1. As these have been calibrated and the model validated by experiments, Sisyphe's results can assist the validation of our model. 
Table 1

Parameter values for the migrating trench test case Villaret et al. (2016)

\begin{tabular}{|l|l|}
\hline Variable Name & Variable Value \\
\hline Length in $x$-direction & $16 \mathrm{~m}$ \\
Length in $y$-direction & $1.1 \mathrm{~m}$ \\
Morphological simulation time & $15 \mathrm{~h}$ \\
Depth & $0.397 \mathrm{~m}$ \\
Downstream elevation & $0.4 \mathrm{~m}$ \\
Upstream flux & $0.22 \mathrm{~m}^{3} \mathrm{~s}^{-1}$ \\
Median particle size $\left(d_{50}\right)$ & $1.6 \times 10^{-4} \mathrm{~m}$ \\
Sediment density $\left(\rho_{s}\right)$ & $2650 \mathrm{~kg} \mathrm{~m}^{-3}$ \\
Water density $\left(\rho_{f}\right)$ & $1000 \mathrm{~kg} \mathrm{~m}^{-3}$ \\
Kinematic viscosity $(v)$ & $1 \times 10^{-6} \mathrm{~m}^{2} \mathrm{~s}^{-1}$ \\
Bed sediment porosity $\left(p^{\prime}\right)$ & 0.4 \\
Diffusivity $\left(\epsilon_{s}\right)$ & $0.01 \mathrm{~m}^{2} \mathrm{~s}^{-1}$ \\
Nikuradse friction height $\left(k_{s}\right)$ & $0.025 \mathrm{~m}^{2}$ \\
\hline
\end{tabular}

Thus, we use the same parameter values in Thetis. We also use a grid with a mesh size of $\Delta x=0.2 \mathrm{~m}$ in the $x$-direction and of $\Delta y=0.22 \mathrm{~m}$ in the $y$-direction. Villaret et al. (2016) use a finer $\Delta y$ of $0.11 \mathrm{~m}$, but we find our Thetis results are consistent with either $\Delta y$, and thus adopt the least computationally expensive option. Finally, we use the boundary conditions from Section 2 and set the incoming suspended sediment flow rate so that the erosion flux, $E_{b}$, equals the deposition flux, $D_{b}$, at the upstream boundary. Hence, we have sediment equilibrium and the bed remains unaltered at the inlet.

\subsection{Results}

In this section, we run both Thetis and Sisyphe for this test case. As discussed in Section 2.5, we first run a pure hydrodynamics simulation for $200 \mathrm{~s}$ ramping up the initial hydrodynamic conditions for our coupled simulation with bedload and suspended sediment transport. We do not use either the slope effect angle correction or secondary current here because both are superfluous in a straight channel.

Figure 2 shows that the bedlevel results from Thetis and Sisyphe agree in both magnitude and profile, but are clearly different from the experimental data. By contrast, when Villaret et al. (2016) use the parameter values in Table 1, the Sisyphe results agree with the experimental data. The difference between the two set-ups is the timestep, $\Delta t$ : Villaret et al. (2016) use $\Delta t=1 \mathrm{~s}$, whereas we use $\Delta t=0.05 \mathrm{~s}$ in Figure 2. This choice of $\Delta t$ is because Thetis requires a smaller Courant number $(U \Delta t / \Delta x)$ than Sisyphe and for comparability reasons the same $\Delta t$ is used in both models. A possible explanation of this Courant number requirement in Thetis is that the overall model can be perceived as semi-implicit since all model equations are solved implicitly, while the coupling of the hydrodynamic and sediment transport components is explicit.

Figure 2 also illustrates that using either a morphological scale factor of 10 or 1 in our Thetis model gives very similar results. Unless otherwise stated all figures in this section are produced using a morphological scale factor of 10. Although Sisyphe has an option for a morphological scale factor, it is not imposed in this work in Sisyphe because neither Villaret et al. (2016) or Villaret et al. (2013) apply it.

\subsubsection{Sensitivity study}

The dependence of Sisyphe's results on $\Delta t$ suggests this test case would benefit from a sensitivity study on the robustness of the models to small changes in physical parameters, timestep and/or mesh step size. First, we explore the impact of varying $\Delta t$ and the mesh size $\Delta x$ on the final bedlevel. Note that the mesh size $\Delta y$ has no effect because there is negligible bedlevel variation in that direction. Figure 3a shows that the Sisyphe bedlevel results vary significantly with $\Delta t$. Only when $\Delta t=1 \mathrm{~s}$, the value of Villaret et al. (2016), is there agreement between Sisyphe and the experimental data. As $\Delta t$ decreases, Sisyphe's results converge to the same result as Thetis in Figure 2. By contrast, bedlevel results from Thetis are largely insensitive to changes in $\Delta t$, as seen in Figure $3 \mathrm{~b}$. Figures $3 \mathrm{~b}, 3 \mathrm{c}$ and $3 \mathrm{~d}$ show how both models are relatively insensitive to changes in $\Delta x$ for this test case.

Thetis produces robust and accurate results for small Courant numbers, but not for large values, unlike Sisyphe (as discussed earlier in this subsection). This prevents us from comparing the two models for every $\Delta x$ and $\Delta t$ combination run in Sisyphe. However, although Sisyphe is stable for larger values of $\Delta t$, the results it produces for these values are not timestep convergent and show significant variability. 


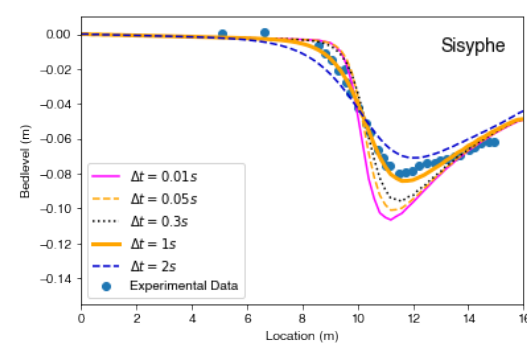

(a) Sisyphe with $\Delta x=0.2 m$.

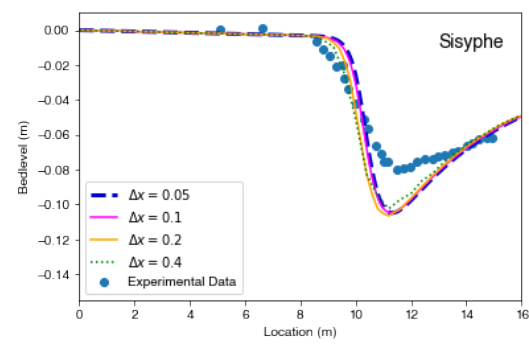

(c) Sisyphe with $\Delta t=0.01 \mathrm{~s}$.

Figure 3: Sensitivity of bedlevel to $\Delta x$ and $\Delta t$.

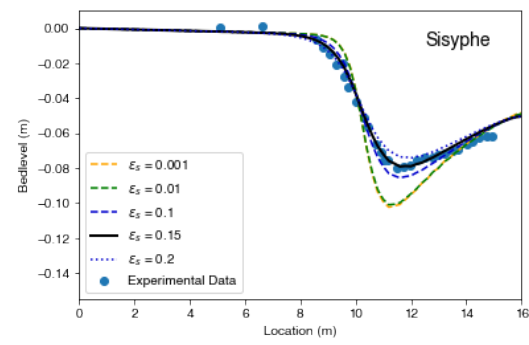

(a) Sisyphe.

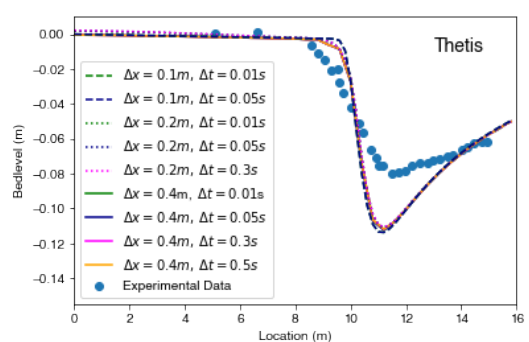

(b) Thetis.

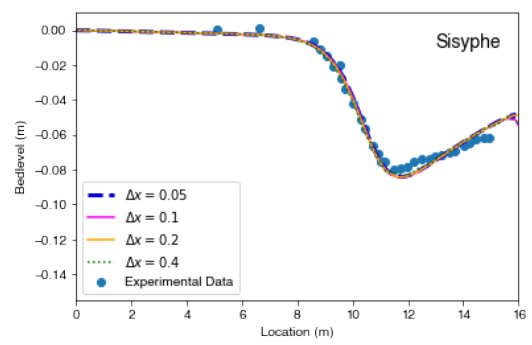

(d) Sisyphe with $\Delta t=1 \mathrm{~s}$.

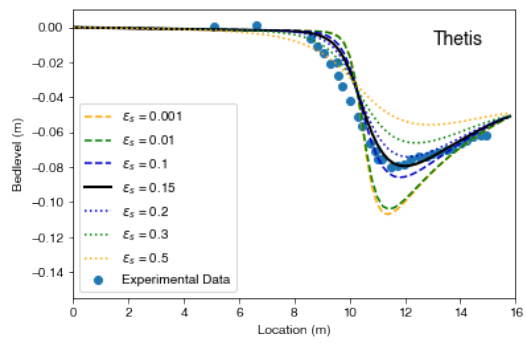

(b) Thetis.

Figure 4: Sensitivity of bedlevel to diffusivity $(\Delta x=0.2 \mathrm{~m}, \Delta t=0.05 \mathrm{~s})$.

Furthermore, we run a small study to investigate whether Sisyphe is always sensitive to $\Delta t$ for this test case. When the method of characteristics is chosen for the hydrodynamics, as in Villaret et al. (2016), we find Sisyphe is always sensitive to $\Delta t$, independent of the choice of morphodynamic scheme. Other methods for the hydrodynamics have stricter Courant number criteria, requiring $\Delta t<0.01 \mathrm{~s}$ to run (even smaller than our Thetis value), meaning this effect is less noticeable.

For small values of $\Delta t$, Thetis and Sisyphe are consistent. We conjecture that the errors caused in Sisyphe with larger $\Delta t$ values manifest themselves as an increase in effective diffusivity in the model. We thus conduct a sensitivity study for the sediment turbulent diffusivity coefficient, $\epsilon_{s}$. For this study, we choose $\Delta t=0.05 \mathrm{~s}$ in both models because Sisyphe is close to convergence for this value, at a relatively small computational time (approximately $50 \mathrm{~min}$ ). Bedlevel results from both Sisyphe and Thetis in Figures $4 \mathrm{a}$ and $4 \mathrm{~b}$ show they are indeed greatly affected by $\epsilon_{s}$ and, importantly, that both models behave consistently. Note that, due to stability constraints, Sisyphe does not run with $\epsilon_{s}>0.2 \mathrm{~m}^{2} \mathrm{~s}^{-1}$, unlike Thetis. The observed sensitivity to $\epsilon_{s}$ is to be expected because the grid Peclet number $\left(U \Delta x / \epsilon_{s}\right)$ decreases with $\epsilon_{s}$, making diffusion the key driver of the sediment concentration equation, rather than advection. Thus, we can use $\epsilon_{s}$ to calibrate both models; in Sisyphe, $\Delta t$ can be used to similar effect.

If we set $\epsilon_{s}=0.15 \mathrm{~m}^{2} \mathrm{~s}^{-1}$, Thetis and Sisyphe's converged results agree well with each other and with the experimental data, as shown clearly in Figure 5. Thus, we have validated Thetis for this simple test case. 


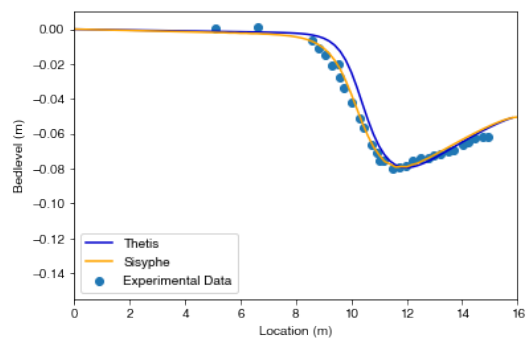

Figure 5: Bedlevel from Thetis and Sisyphe after $15 \mathrm{~h}$ using $\epsilon_{s}=0.15 \mathrm{~m}^{2} / \mathrm{s}$ and $\Delta t=0.05 \mathrm{~s}$.

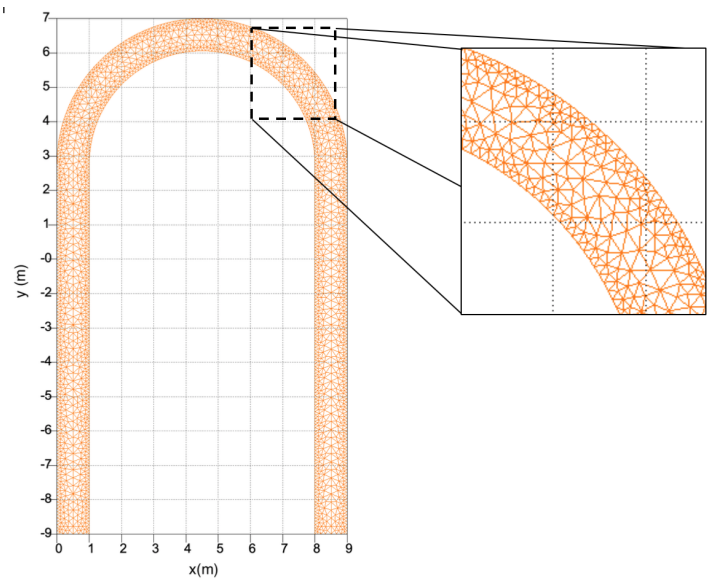

Figure 6: Meander mesh and domain used both in Thetis and Sisyphe by Villaret et al. (2013).

\section{Meander test case}

Our second test case regards the curved channel of a meander, which requires and demonstrates the implementation of a slope effect angle correction and a secondary current. This test case is used to validate these additional functionalities, and affirm our model can handle more complex and realistic set-ups.

\subsection{Test case configuration}

We use the configuration from experiment 4 from Yen and Lee (1995) and validate Thetis through the experimental data and Sisyphe results from Villaret et al. (2013). Most of the bed changes occur at the boundary so, following Villaret et al. (2013), we use a finer mesh there $(0.1 \mathrm{~m})$ and a coarser one $(0.25 \mathrm{~m})$ along the centre of the channel, as in Figure 6.

We impose time dependent flux and elevation boundary conditions reproducing Yen and Lee (1995). The initial inflow flux and outflow elevation are $0.02 \mathrm{~m}^{3} \mathrm{~s}^{-1}$ and $0 \mathrm{~m}$, respectively. Both increase linearly until reaching their respective maximums of $0.053 \mathrm{~m}^{3} \mathrm{~s}^{-1}$ and $0.103 \mathrm{~m}$ at $100 \mathrm{~min}$, and then decrease linearly to their initial values at $5 \mathrm{~h}$. We also impose a free-slip condition on the meander boundary walls.

In both Thetis and Sisyphe, we use the parameter values summarised in Table 2. Following Villaret et al. (2013), we only model bedload transport because this is the principal sediment transport component in rivers. Hence, we do not need to specify the diffusivity coefficient $\epsilon_{s}$. The implementation of the secondary current requires we determine the flow roughness to set the value of $\alpha^{\prime}$ in Eq. (33). Following Kulkarni and Sahoo (2013), we calculate that the roughness Reynolds number, $\left(k_{s} \sqrt{\tau_{b}}\right) /\left(v \sqrt{\rho_{f}}\right)$, is approximately 80 , and conclude we are in a rough turbulent flow regime. Consistently with Tassi and Villaret (2014), we use $\alpha^{\prime}=0.75$.

\subsection{Results}

\subsubsection{Modelling the hydrodynamics}

If we use the same viscosity value when modelling the hydrodynamics for the meander test case as for the migrating trench test case $\left(1 \times 10^{-6} \mathrm{~m}^{2} \mathrm{~s}^{-1}\right)$, we find that our model does not accurately solve the flow at the meander boundary 
Table 2

Parameter values for the meander test case Villaret et al. (2013)

\begin{tabular}{|l|l|}
\hline Variable Name & Variable Value \\
\hline Channel width & $1 \mathrm{~m}$ \\
Inner radius & $3.5 \mathrm{~m}$ \\
Outer radius & $4.5 \mathrm{~m}$ \\
Straight reach at channel ends & $11.5 \mathrm{~m}$ \\
Morphological simulation time & $5 \mathrm{~h}$ \\
Depth & $0.0544 \mathrm{~m}$ \\
Median particle size $\left(d_{50}\right)$ & $1 \times 10^{-3} \mathrm{~m}$ \\
Sediment density $\left(\rho_{s}\right)$ & $2650 \mathrm{~kg} \mathrm{~m}^{-3}$ \\
Water density $\left(\rho_{f}\right)$ & $1000 \mathrm{~kg} \mathrm{~m}^{-3}$ \\
Kinematic viscosity $(v)$ & $0.01 \mathrm{~m}^{2} \mathrm{~s}^{-1}$ \\
Bed sediment porosity $\left(p^{\prime}\right)$ & 0.4 \\
Nikuradse friction height $\left(k_{s}\right)$ & $0.0035 \mathrm{~m}$ \\
\hline
\end{tabular}

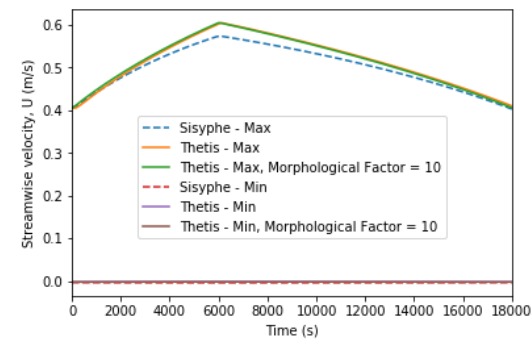

(a) Streamwise velocity, $\bar{u}_{1}$.

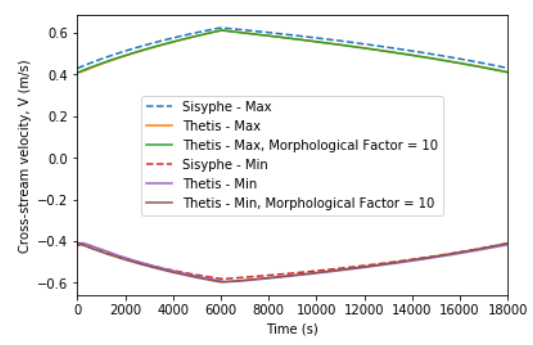

(b) Cross-stream velocity, $\bar{u}_{2}$.

Figure 7: Minimum and maximum velocities from Thetis $\left(v=0.035 \mathrm{~m}^{2} \mathrm{~s}^{-1}\right.$, slope $\left.=0.0035\right)$ with a morphological scale factor of 1 and 10, and Sisyphe $\left(v=0.01 \mathrm{~m}^{2} \mathrm{~s}^{-1}\right.$, slope $\left.=0.002\right)$.

walls. Instead of finding a smooth solution, the flow magnitude increases dramatically in the cells closest to the boundary. However molecular viscosity values $\left(1 \times 10^{-6} \mathrm{~m}^{2} \mathrm{~s}^{-1}\right)$ only become relevant at the Kolmogorov scale. Our test case is at a much larger scale where a viscous force exists caused by turbulence, Thus the viscosity is a turbulence or eddy viscosity and a value of $1 \times 10^{-3} \mathrm{~m}^{2} \mathrm{~s}^{-1}$ (the value by Vouriot et al. (2019) for their Thetis test case) is more appropriate. As we increase $v$ in the hydrodynamic equations (1) and (2) the flow becomes smoother and for viscosity values of $O\left(1 \times 10^{-3}\right)$ the boundary issue no longer exists. The issue itself is related to how boundary conditions at closed impermeable boundaries are imposed in equal order DG discretisations and will be addressed in the future.

As we are not using a turbulence model, to find the correct value of $v$, we use Sisyphe's hydrodynamic results to calibrate our model, noting that Villaret et al. (2013) use $1 \times 10^{-2} \mathrm{~m}^{2} \mathrm{~s}^{-1}$. These alterations in $v$ change the nature of the test case, but can be balanced by altering the longitudinal bed slope. In Yen and Lee (1995), the meander has a longitudinal bed slope of 0.002, as in Villaret et al. (2013). We find that if we set the longitudinal bed slope to 0.0035 and $v$ to $0.035 \mathrm{~m}^{2} \mathrm{~s}^{-1}$, our model's velocities match those in Sisyphe reasonably well, as shown in Figures $7 \mathrm{a}$ and $7 \mathrm{~b}$. These figures also show that even with time dependent boundary conditions, using a morphological scale factor equal to either 1 or 10 in Thetis gives equivalent results. Thus, unless otherwise stated, in this section our Thetis results are produced with a morphological scale factor of 10 .

\subsubsection{Modelling sediment transport}

As the hydrodynamic flow of Thetis agrees with Sisyphe, we introduce sediment transport into the models. As discussed in Section 2.5, we first run a simulation for $200 \mathrm{~s}$ solving only the hydrodynamics with a fixed flux inflow of $0.02 \mathrm{~m}^{3} \mathrm{~s}^{-1}$ and outflow elevation of $0 \mathrm{~m}$. For our full sediment transport simulation, we use these results as the initial hydrodynamic conditions and impose time dependent flux and elevation conditions from Section 5.1 as the boundary conditions. We present the scaled bedlevel evolution results which is defined as

$$
\text { Scaled Bedlevel Evolution }=\frac{z_{\text {final }}-z_{\text {initial }}}{z_{\text {initial }}}
$$

where $z_{\text {final }}$ is the final bedlevel after $5 \mathrm{~h}$ and $z_{\text {initial }}$ is the initial bedlevel of $-0.0544 \mathrm{~m}$. 


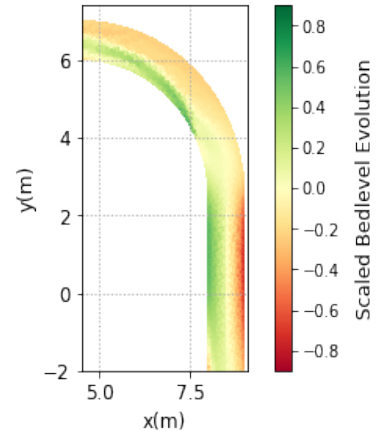

(a) No physical corrections.

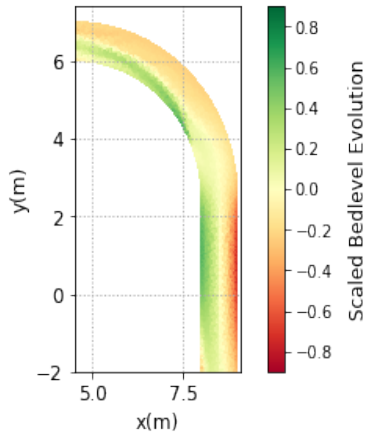

(b) Only slope effect magnitude.

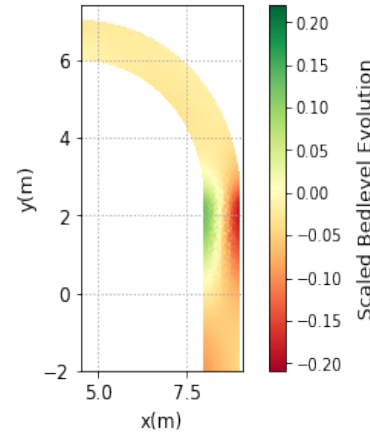

(c) Both slope effect corrections (note smaller colour scale).

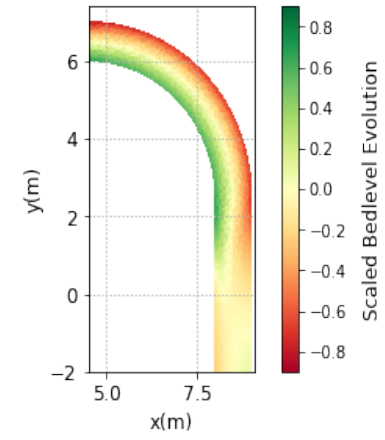

(d) All physical corrections.

Figure 8: Meander section showing scaled bedlevel evolution from Thetis with different physical corrections to $\mathbf{Q}_{\mathbf{b}}$.

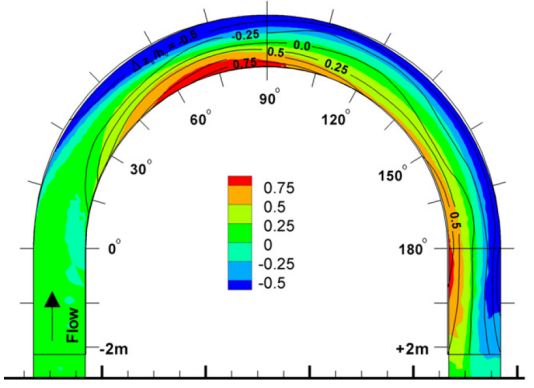

Figure 9: Scaled bedlevel evolution from Sisyphe (coloured bars) and experimental data (black contours). Source: Villaret et al. (2013).

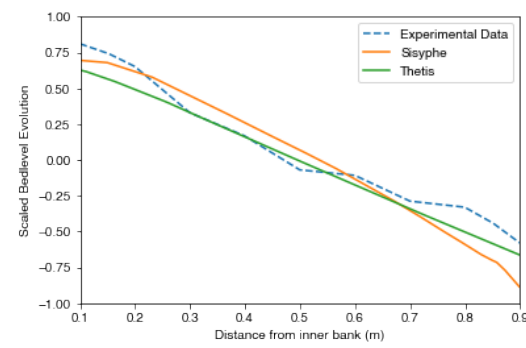

(a) Cross-section at $90^{\circ}$.

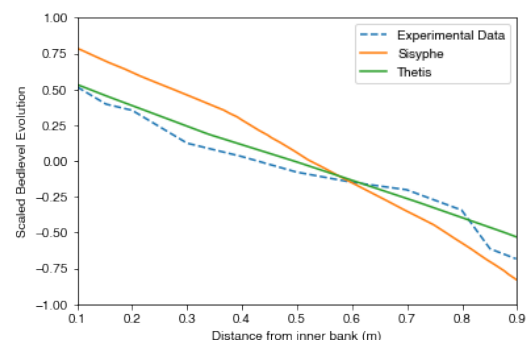

(b) Cross-section at $180^{\circ}$.

Figure 10: Scaled bedlevel evolution from Thetis (with $v=0.035 \mathrm{~m}^{2} \mathrm{~s}^{-1}$, slope $=0.0035$ ), Sisyphe Villaret et al. (2013) and experimental data Yen and Lee (1995).

Figure 8 shows the effects of implementing secondary current and slope effects on the bedlevel evolution at the meander outflow. The slope effect magnitude correction has little effect compared to the secondary current and slope effect angle corrections, likely because the slopes in this test case are fairly gentle.

In Figure 8d, the final scaled bedlevel evolution result is shown, with erosion at the outer bend and deposition at the inner bed, as expected from physical intuition. Comparing this figure with Figure 9, we see Thetis result has the same distribution and magnitude as the experiment and Sisyphe. Hereafter, unless otherwise stated, all Sisyphe results are those presented in Villaret et al. (2013).

To compare our Thetis result with the experiment and Sisyphe's results more accurately, we take a cross-section at the $90^{\circ}$ and $180^{\circ}$ angles marked on Figure 9. Figures 10a and 10b show our model approximates the experimental results better than Sisyphe, with a particular improvement at the $180^{\circ}$ cross-section and in the bedlevel erosion at both cross-sections. 
Table 3

Sum of relative error norms for different values of longitudinal slope and $v\left(\mathrm{~m}^{2} \mathrm{~s}^{-1}\right)$.

\begin{tabular}{|l|c|c|c|c|}
\hline Slope & $\boldsymbol{v}=\mathbf{0 . 0 2 5}$ & $\boldsymbol{v}=\mathbf{0 . 0 3 5}$ & $\boldsymbol{v}=\mathbf{0 . 0 5}$ & $\boldsymbol{v}=\mathbf{0 . 0 7 5}$ \\
\hline $\mathbf{0 . 0 0 3}$ & 0.5041 & 0.4934 & 0.4847 & 0.4930 \\
\hline $\mathbf{0 . 0 0 3 5}$ & 0.4911 & 0.4828 & 0.4752 & 0.4851 \\
\hline $\mathbf{0 . 0 0 4}$ & 0.5253 & 0.5167 & 0.5106 & 0.5199 \\
\hline $\mathbf{0 . 0 0 4 5}$ & 0.5809 & 0.5707 & 0.5635 & 0.5686 \\
\hline
\end{tabular}

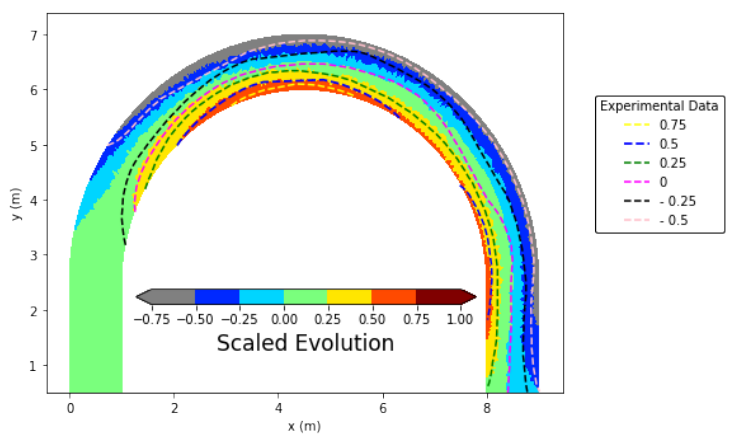

Figure 11: Scaled bedlevel evolution from Thetis with $v=0.05 \mathrm{~m}^{2} \mathrm{~s}^{-1}$, slope $=0.0035$ and experimental data Yen and Lee (1995).

\subsubsection{Calibration study}

In Section 5.2.1, we used the hydrodynamic results from Sisyphe to calibrate the viscosity and longitudinal slope in Thetis because those from the experiment are not available. However, Figures 10a and 10b show Sisyphe does not agree completely with the experimental data. Hence, to improve our model's accuracy, we re-run the calibration study this time using the experimental data as the 'real solution'. We seek to minimize the relative error norm at both the $90^{\circ}$ and $180^{\circ}$ cross-section and thus minimise

$$
\frac{\left\|y_{90_{i}}-y_{90_{i}}\right\|_{2}}{\left\|y_{90_{i}}\right\|_{2}}+\frac{\left\|y_{180_{i}}-y_{180_{i}}\right\|_{2}}{\left\|y_{180_{i}}\right\|_{2}},
$$

where $\hat{y_{\cdot}}$ is the experimental data and $y_{\cdot_{i}}$ our model result. The results are given in Table 3 . Hence, we conclude that a viscosity of $0.05 \mathrm{~m}^{2} \mathrm{~s}^{-1}$ and a longitudinal slope of 0.0035 yield the best approximation to the experimental data. For these values, Figure 11 shows that the scaled bedlevel evolution from Thetis agrees closely with the experiment, particularly at the inner bend and at the meander outflow. Comparing the experiment, Sisyphe results in Figure 9 and our results in Figure 11, we see that Thetis predicts the bedlevel erosion to a greater degree of accuracy, particularly at the outer bend. Furthermore, it shows uniform erosion at the inflow bedlevel, unlike Sisyphe (Figure 9), although neither model predicts the inflow bedlevel particularly accurately.

For rigour, we run Sisyphe with these optimised values for viscosity and longitudinal slope. The resulting bedlevel change is shown in Figures $12 \mathrm{a}$ and $12 \mathrm{~b}$ at the $90^{\circ}$ and $180^{\circ}$ cross-sections, respectively. There is a slight improvement in the values of the total relative error norm (46), which falls from 1.144 for the results from Villaret et al. (2013) to 1.067 for the optimised values. However, the errors of Sisyphe are still higher than those obtained for Thetis.

\subsubsection{Sensitivity Study}

Given Sisyphe's sensitivity to $\Delta t$ discussed in Section 4.2.1, we conduct a sensitivity study on $\Delta t$ and the mesh step size, $\Delta x$, maintaining the ratio between the fine and coarse meshes at 2:5. We run Sisyphe and use our optimised viscosity and longitudinal slope values for consistency with Thetis.

Figure 13b shows Thetis is insensitive to $\Delta t$, whereas Sisyphe in Figure 13a is sensitive to $\Delta t$, as in the migrating trench test case. Although for $\Delta t \leq 0.25 \mathrm{~s}$ Sisyphe's results are robust, for larger $\Delta t$ values they are both sensitive and inaccurate. Furthermore, for this test case, Thetis converges for $\Delta t<10 \mathrm{~s}$, meaning it is much less computationally expensive than Sisyphe, which requires $\Delta t \leq 0.25 \mathrm{~s}$.

Figures $13 \mathrm{c}$ and $13 \mathrm{~d}$ show both models are relatively insensitive to the mesh step size $\Delta x$. There are slight differences when a fine $\Delta x=0.25 \mathrm{~m}$ is used in both models, suggesting our choice of a fine $\Delta x=0.1 \mathrm{~m}$ is appropriate. 


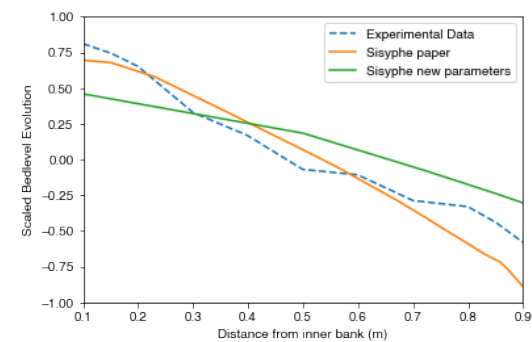

(a) Cross-section at $90^{\circ}$

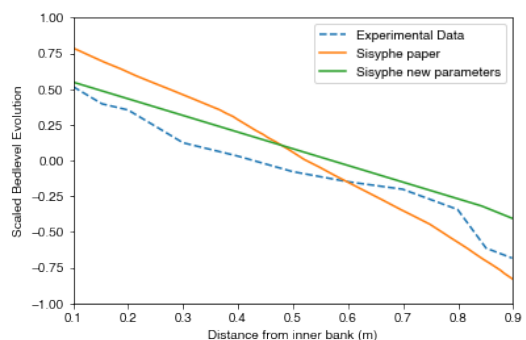

(b) Cross-section at $180^{\circ}$.

Figure 12: Scaled bedlevel evolution from Sisyphe in Villaret et al. (2013), Sisyphe with $v=0.05 \mathrm{~m}^{2} \mathrm{~s}^{-1}$ and slope $=0.0035$, and experimental data Yen and Lee (1995).

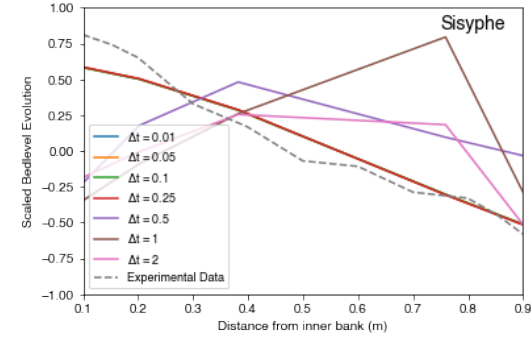

(a) Sisyphe to $\Delta t$.

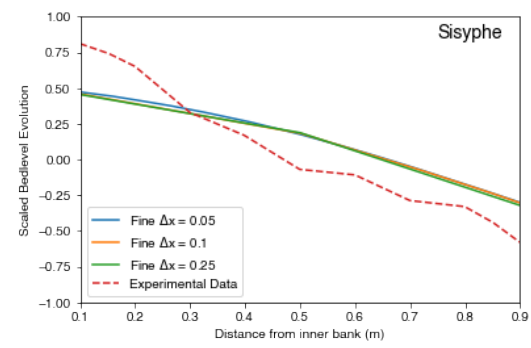

(c) Sisyphe to $\Delta x$.

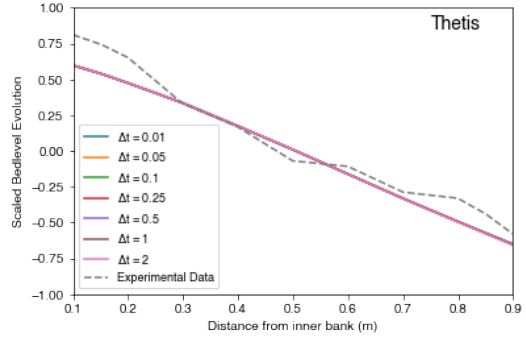

(b) Thetis to $\Delta t$.

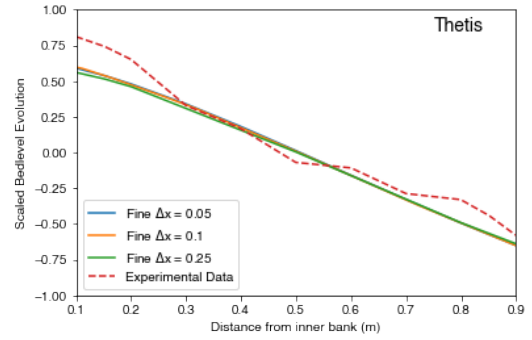

(d) Thetis to $\Delta x$.

Figure 13: Sensitivity of bedlevel to $\Delta x$ and $\Delta t$ (90 cross-section).

As in Section 4.2, we assess whether Sisyphe results depend on the discretisation of the advection terms. Our preliminary results show that Sisyphe's sensitivity to $\Delta t$ is independent of the choice of morphodynamic scheme, as indicated in the previous example. The strict Courant number stability criteria of other Sisyphe hydrodynamic discretisations means they require small $\Delta t$ to run and thus the effect is less noticeable.

Finally, Figures $14 \mathrm{a}$ and $14 \mathrm{~b}$ provide an overview of our results and show not only that we have validated our model, but that it is more accurate than Sisyphe for this more complex test case. Figures 14a and 14b also confirm that a morphological scale factor of 10 is appropriate because there is no observable difference between our results with a morphological scale factor of 10 and 1 (i.e. no scaling).

\section{Benchmarking}

Finally, we compare the computational times of Thetis and Sisyphe for both the test cases discussed in this work and summarise the results in Table 4. Note that for Sisyphe we have chosen the most efficient matrix storage method following guidance in Lang et al. (2014). When the same $\Delta t$ and morphological scale factor are used in both models, Thetis is slower, partly since on the same mesh a DG discretisation possesses significantly more degrees of freedom. However, the robustness we get from Thetis's DG based discretisation allows us to obtain accurate results with larger $\Delta t$ values and the use of a morphological scale factor. If we take advantage of this, for the simple migrating trench test case, Thetis can be made competitive with Sisyphe and for the more complex meander test case the computational 


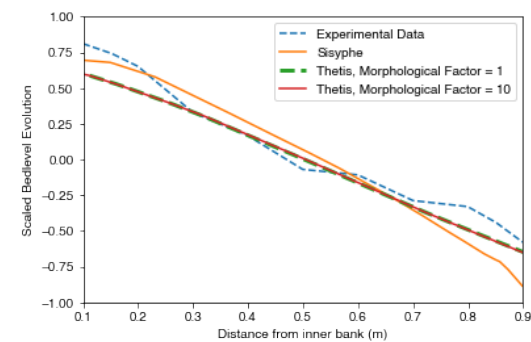

(a) Cross-section at $90^{\circ}$.

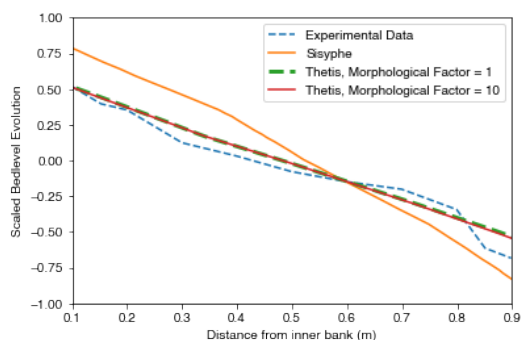

(b) Cross-section at $180^{\circ}$.

Figure 14: Scaled bedlevel evolution from Thetis $\left(v=0.05 \mathrm{~m}^{2} \mathrm{~s}^{-1}\right.$ and slope $\left.=0.0035\right)$ with a morphological scale factor of 1 and 10, Sisyphe Villaret et al. (2013) and experimental data Yen and Lee (1995).

\section{Table 4}

Comparison of computational time (seconds). For the migrating trench, $\Delta t=0.05 \mathrm{~s}$ and increased $\Delta t=0.3 \mathrm{~s}$; for the meander $\Delta t=0.1 \mathrm{~s}$ and increased $\Delta t=10 \mathrm{~s}$.

\begin{tabular}{|c|c|c|c|c|}
\hline & Sisyphe & Thetis & $\begin{array}{c}\text { Thetis (scale } \\
\text { factor) }\end{array}$ & $\begin{array}{c}\text { Thetis } \\
\text { (scale factor, } \\
\text { increased } \Delta t \text { ) }\end{array}$ \\
\hline $\begin{array}{c}\text { Migrating } \\
\text { Trench }\end{array}$ & 3,427 & 341,717 & 39,955 & 12,422 \\
\hline Meander & 980 & 60,784 & 10,811 & 1,212 \\
\hline
\end{tabular}

time is very similar. In Table 4, we use a moderate morphological scale factor of 10, but if we use a morphological scale factor of 50, for the meander test case Thetis takes less than half the time that Sisyphe does, with only very slight observable differences compared to with a morphological scale factor of 1 . This is likely because for the simple test case, we have the disadvantages of the additional computatonal cost of DG without experiencing the advantages in robustness that DG exhibits for more complex cases.

\section{Conclusion}

In this work, we have presented a new 2D depth-averaged coupled hydrodynamic and sediment transport functionality within the finite element based coastal ocean model Thetis. Our model makes significant, novel contributions to the complex problem of modelling sediment transport. It is shown to be both accurate and stable, as well as comparable in computational cost to other standard models. To the best of our knowledge, it is the first full morphodynamic model employing a DG based discretisation. We report on several new capabilities within Thetis, including bedload transport, bedlevel changes, slope effect corrections, a secondary current correction, a sediment transport source term, a velocity correction factor in the sediment concentration equation, and a morphological scale factor. All these were validated using the migrating trench and meander test cases, indicating the significance of each of the additional components. The coupled and nonlinear nature of the problem makes this type of model very sensitive to parameter changes. However, Thetis is found to be largely insensitive to changes in timestep and mesh grid size, unlike the current state-of-the-art model Sisyphe, which is found to have a much larger variability, particularly with respect to the timestep in the case of the test cases considered in this work. In future work, we will use our model in a coastal zone case study requiring coupled wave and current modelling, and will further consider adjoint-based model calibration and the use of mesh adaptivity.

\section{Acknowledgements}

The authors thank Dr Catherine Villaret for her help in the set-up of Sisyphe. MCAC's work was funded through the EPSRC CDT in Mathematics for Planet Earth. AA acknowledges the support of NERC through the fellowship grant NE/R013209/2. MDP, JRP and CJC acknowledge the support of EPSRC through the grants EP/R029423/1 and $\mathrm{EP} / \mathrm{R} 007470 / 1$. 


\section{Computer code availability}

The relevant Thetis code for the morphodynamic model presented in this work can be found at https://github. com/mc4117/morphodynamic_model.

\section{References}

Amoudry, L.O., 2008. A review on coastal sediment transport modelling. Proudman Oceanographic Laboratory, Liverpool, UK.

Amoudry, L.O., Souza, A.J., 2011. Deterministic coastal morphological and sediment transport modeling: A review and discussion. Reviews of Geophysics 49.

CIESIN, 2013. Low Elevation Coastal Zone (LECZ) Urban-Rural Population and Land Area Estimates, Version 2. NASA Socioeconomic Data and Applications Center (SEDAC), Palisades, New York, USA. URL: https://doi .org/10.7927/H4MW2F2J.

Danilov, S., 2013. Ocean modeling on unstructured meshes. Ocean Modelling 69, 195-210.

Dawson, C., Sun, S., Wheeler, M.F., 2004. Compatible algorithms for coupled flow and transport. Computer Methods in Applied Mechanics and Engineering 193, 2565-2580.

Deltares, 2014. Delft3D-FLOW Simulation of multi-dimensional hydrodynamic flows and transport phenomena including sediments. User Manual. Delft, The Netherlands. URL: https://oss.deltares.nl/documents/183920/185723/Delft3D-FLOW_User_Manual.pdf.

Engelund, F., 1974. Flow and bed topography in channel beds. Journal of the Hydraulics Division 100.

Epshteyn, Y., Rivière, B., 2007. Estimation of penalty parameters for symmetric interior penalty Galerkin methods. Journal of Computational and Applied Mathematics 206, 843-872.

Farrell, P.E., Ham, D.A., Funke, S.W., Rognes, M.E., 2013. Automated derivation of the adjoint of high-level transient finite element programs. SIAM Journal on Scientific Computing 35, C369-C393.

Garcia, M., Parker, G., 1991. Entrainment of bed sediment into suspension. Journal of Hydraulic Engineering 117, 414-435.

Gerritsen, H., de Goede, E., Platzek, F., van Kester, J., Genseberger, M., Uittenbogaard, R., 2008. Validation Document Delft3D-FLOW; a software system for 3D flow simulations. Technical Report. Deltares. Delft, The Netherlands.

Hervouet, J.M., 1999. TELEMAC, a hydroinformatic system. Houille Blanche-revue Internationale De L Eau 54, 21-28. doi:10.1051/1hb/ 1999029.

Hervouet, J.M., 2007. Hydrodynamics of Free Surface Flows, Modelling with the Finite-element Method. John Wiley \& Sons Ltd, West Sussex, UK.

Huybrechts, N., Villaret, C., Hervouet, J.M., 2010. Comparison between 2D and 3D modelling of sediment transport: Application to the dune evolution, in: Proceedings of the 5th International Conference on fluvial Hydraulics, Braunschweig, Germany.

Kärnä, T., Kramer, S., Mitchell, L., Ham, D., Piggott, M., Baptista, A., 2018. Thetis coastal ocean model: discontinuous Galerkin discretization for the three-dimensional hydrostatic equations. Geoscientific Model Development 11, 4359-4382.

Kubatko, E.J., Westerink, J.J., Dawson, C., 2006. An unstructured grid morphodynamic model with a discontinuous Galerkin method for bed evolution. Ocean modelling 15, 71-89.

Kulkarni, V., Sahoo, N., 2013. Module 5: Viscous Incompressible flow; Lecture 5 : Internal Flow - Part IV. https://nptel .ac.in/courses/ 101103004/module5/lec5/4.html.

Landsberg, A., Chtchelkanova, A., Lind, C., Boris, J., Young, T., 1998. Fast3D user and programmer reference manual. US Naval Research Laboratory. Washington DC, USA.

Lang, P., Desombre, J., ATA, R., Goeury, C., Hervouet, J.M., 2014. TELEMAC-2D modelling system - User manual.

Leveque, R.J., 1996. High-resolution conservative algorithms for advection in incompressible. SIAM Journal on Numerical Analysis $33,627-665$.

Li, B.Q., 2006. Discontinuous Finite Elements in Fluid Dynamics and Heat Transfer. Springer Science \& Business Media, Berlin, Germany.

McManus, T., Percival, J., Yeager, B., Barral, N., Gorman, G., Piggott, M., 2017. Moving mesh methods in Fluidity and Firedrake. Technical Report July. doi:10.13140/RG.2.2.27670.24648.

Michoski, C., Dawson, C., Mirabito, C., Kubatko, E.J., Wirasaet, D., Westerink, J.J., 2013. Fully coupled methods for multiphase morphodynamics. Advances in Water Resources 59, 95-110. URL: http://dx.doi.org/10.1016/j.advwatres. 2013.05.002, doi:10.1016/j . advwatres.2013.05.002.

Pan, W., Kramer, S.C., Piggott, M.D., 2019. Multi-layer non-hydrostatic free surface modelling using the discontinuous Galerkin method. Ocean Modelling 134, 68-83.

Papanicolaou, A.T.N., Elhakeem, M., Krallis, G., Prakash, S., Edinger, J., 2008. Sediment transport modeling review - current and future developments. Journal of Hydraulic Engineering 134, 1-14.

Park, S.W., Ahn, J., 2019. Experimental and numerical investigations of primary flow patterns and mixing in laboratory meandering channel. Smart Water 4, 4. URL: https: //doi.org/10.1186/s40713-019-0016-y, doi:10.1186/s40713-019-0016-y.

Rathgeber, F., Ham, D.A., Mitchell, L., Lange, M., Luporini, F., McRae, A.T., Bercea, G., Markall, G., Kelly, P.H., 2017. Firedrake: automating the finite element method by composing abstractions. ACM Transactions on Mathematical Software (TOMS) 43, 24.

Roelvink, D., Van Dongeren, A., McCall, R., Hoonhout, B., Van Rooijen, A., Van Geer, P., De Vet, L., Nederhoff, K., Quataert, E., 2015. XBeach Technical Reference: Kingsday Release. Delft, The Netherlands: Deltares, Technical report .

Segur, H., 2009. Lecture 8 : The Shallow-Water Equations. Woods Hole Oceanographic Institution, Woods Hole, Massachusetts, USA.

Soulsby, R., 1997. Dynamics of marine sands, a manual for practical applications. Thomas Telford, London, UK.

Syvitski, J., Slingerland, R., Burgess, P., Meiburg, E., Murray, A.B., Wiberg, P., Tucker, G., Voinov, A., 2010. Morphodynamic Models: An Overview. River, Coastal and Estuarine Morphodynamics, RCEM 2009 , 3-20.

Talmon, A., Struiksma, N., Mierlo, M.V., 1995. Laboratory measurements of the direction of sediment transport on transverse alluvial-bed slopes. Journal of Hydraulic Research 33, 495-517. 
Tassi, P., Rhebergen, S., Vionnet, C., Bokhove, O., 2008. A discontinuous Galerkin finite element model for river bed evolution under shallow flows. Computer Methods in Applied Mechanics and Engineering 197, 2930-2947.

Tassi, P., Villaret, C., 2014. Sisyphe v6.3 User's Manual. EDF R\&D. Chatou, France.

Van Rijn, L.C., 1980. Storm surge barrier Oosterschelde-computation of siltation in dredged trenches: Semi-empirical model for the flow in dredged trenches. Deltares, Delft, The Netherlands.

Van Rijn, L.C., 1984. Sediment Transport, Part II: Suspended Load Transport. Journal of Hydraulic Engineering 110, $1613-1641$.

Villaret, C., Hervouet, J.M., Kopmann, R., Merkel, U., Davies, A.G., 2013. Morphodynamic modeling using the Telemac finite-element system. Computers \& Geosciences 53, 105-113.

Villaret, C., Kopmann, R., Wyncoll, D., Riehme, J., Merkel, U., Naumann, U., 2016. First-order uncertainty analysis using Algorithmic Differentiation of morphodynamic models. Computers \& geosciences 90, 144-151.

Vouriot, C.V.M., Angeloudis, A., Kramer, S.C., Piggott, M.D., 2019. Fate of large-scale vortices in idealized tidal lagoons. Environmental Fluid Mechanics 19, 329-348.

Warner, J.C., Sherwood, C.R., Signell, R.P., Harris, C.K., Arango, H.G., 2008. Development of a three-dimensional, regional, coupled wave, current, and sediment-transport model. Computers \& Geosciences 34, 1284-1306.

Warren, I., Bach, H., 1992. MIKE 21: a modelling system for estuaries, coastal waters and seas. Environmental Software 7, $229-240$.

Wu, W., 2007. Computational river dynamics. CRC Press, Boca Raton, Florida, USA.

Yen, C.1., Lee, K.T., 1995. Bed topography and sediment sorting in channel bend with unsteady flow. Journal of Hydraulic Engineering 121, 591-599. 